\title{
Automatic Detection of Blood versus non-Blood Regions on Intravascular Ultrasound (IVUS) Images Using Wavelet Packet Signatures
}

\author{
Amin Katouzian $^{1}$, Babak Baseri ${ }^{3}$, Elisa E. Konofagou ${ }^{1,2}$, Andrew F. Laine ${ }^{1,2}$ \\ Departments of ${ }^{1}$ Biomedical Engineering, and ${ }^{2}$ Radiology of Columbia University, New York, USA \\ ${ }^{3}$ School of Biomedical Sciences, University of Medicine and Dentistry of New Jersey, Newark, USA \\ ak2432@columbia.edu
}

\begin{abstract}
Intravascular ultrasound (IVUS) has been proven a reliable imaging modality that is widely employed in cardiac interventional procedures. It can provide morphologic as well as pathologic information on the occluded plaques in the coronary arteries. In this paper, we present a new technique using wavelet packet analysis that differentiates between blood and non-blood regions on the IVUS images. We utilized the multi-channel texture segmentation algorithm based on the discrete wavelet packet frames (DWPF). A k-mean clustering algorithm was deployed to partition the extracted textural features into blood and non-blood in an unsupervised fashion. Finally, the geometric and statistical information of the segmented regions was used to estimate the closest set of pixels to the lumen border and a spline curve was fitted to the set. The presented algorithm may be helpful in delineating the lumen border automatically and more reliably prior to the process of plaque characterization, especially with $40 \mathrm{MHz}$ transducers, where appearance of the red blood cells renders the border detection more challenging, even manually. Experimental results are shown and they are quantitatively compared with manually traced borders by an expert. It is concluded that our two dimensional (2-D) algorithm, which is independent of the cardiac and catheter motions performs well in both in-vivo and in-vitro cases.
\end{abstract}

Keywords: Intravascular ultrasound, Wavelet Packet Frames, Feature Extraction, Classification.

\section{1- INTRODUCTION}

The number of cardiac intervention procedures supplemented with intravascular ultrasound (IVUS) imaging has been significantly increased due to its clinical applicability and reliability. Although the primary use of the IVUS is taking the cross-sectional images of arterial wall and stenosis, it has been shown that the spectral analysis of backscattered IVUS signals can also be employed for atherosclerotic tissue characterization ${ }^{1-3}$. The most significant plaques are the ones that are soft and rupture-prone, characterized by a thin superficial fibrotic cap with a large underlying necrotic core that may contain thrombosis or nodular calcium ${ }^{4}$. Keeping this in mind, it is reasonable to conclude that the ultimate goal of atherosclerotic tissue characterization is the algorithmic detection of plaque compositions especially rupture-prone plaques, which are ultimately identified by an expert that assesses the classified tissue color maps. Nevertheless, none of the existing algorithms is able to differentiate between the blood cavity and plaques. In other words, the developed algorithms for atherosclerotic tissue characterization cannot be directly deployed to detect the lumen border. For this reason, image processing techniques are used to extract the lumen border along with vessel wall but they fail to classify tissues $^{5,6,7}$.

In this paper, the texture segmentation algorithm based on discrete wavelet packet frame (DWPF) and two-dimensional (2-D) envelope detection introduced by Laine et $\mathrm{al}^{8}$ is used to detect the lumen border in an unsupervised fashion. In tissue characterization, we previously demonstrated that the extracted textural features were perfectly suited for classification and capture characteristics of the plaque with the highest correlation to histology ${ }^{10}$. On the other hand, our results show that the same features can be utilized to detect the blood regions in-vitro and in-vivo on the IVUS images.

Medical Imaging 2008: Ultrasonic Imaging and Signal Processing, edited by Stephen A. McAleavey, Jan D'hooge, Proc. of SPIE Vol. 6920,69200H, (2008) · 1605-7422/08/\$18 · doi: 10.1117/12.773600 


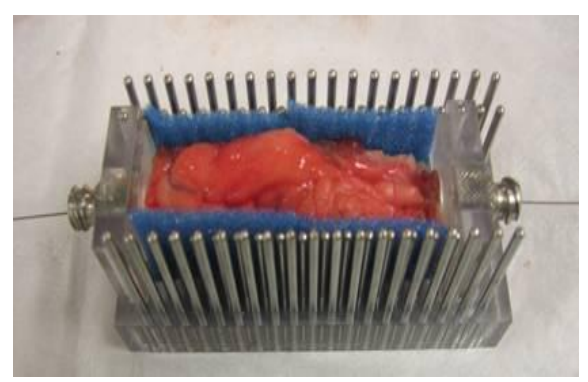

(a)

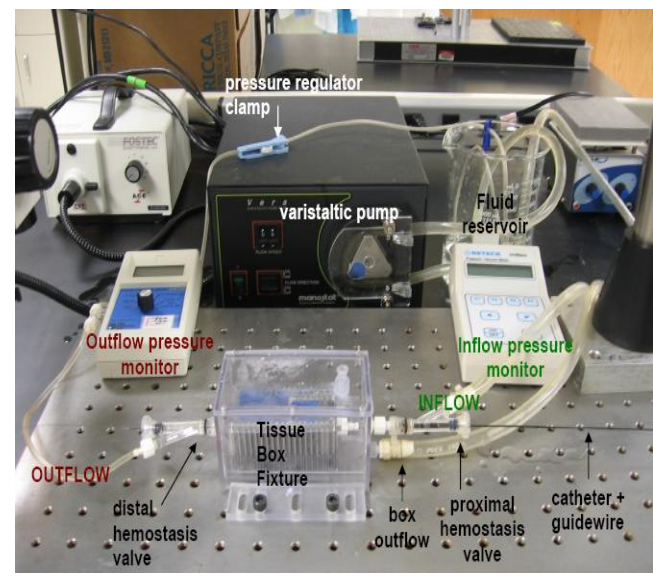

(b)

Figure1. Tissue cage fixture (a), experiment set up (b).

System specifications, specimen preparation and data collection methodologies are introduced in Section 2. We review the wavelet packet representation and describe the method of filter selection and feature extraction in Section 3. In Section 4, we present the classification algorithm along with experimental results and discuss the performance, advantages and limitations of the algorithm. A summary and conclusions are provided in Section 5.

\section{2- SPECIMEN PREPARATION AND DATA COLLECTION}

Acquisition of cross-sectional ultrasound images of right coronary arteries (RCA), left anterior descending (LAD) and left circumflex (LCX) coronary arteries were performed using a $40 \mathrm{MHz}$ rotating single-element Boston Scientific (Fremont CA) transducer. The radiofrequency (RF) data were continuous-time, real-valued and band-limited signals, $x(t)$, and were digitized $x\left(n T_{s}\right)=x[n]=x_{n}$ at periodic time intervals, $T_{s}=2.5 n s\left(f_{s}=400 \mathrm{MHz}\right)$, with two 12-bit Acqiris boards [Monroe, NY]. The catheter pullback speed was $0.5 \mathrm{~mm} / \mathrm{s}$ and the frame rate 30 frames/s. Each raw frame contains 256 lines with 2048 samples per line. For in-vitro data, we collected hearts from two sources: autopsy and transplant surgery, within 24 hours postmortem. The arteries were dissected from the heart with an approximate size of $20-60 \times 20 \times 20 \mathrm{~mm}(l \times w \times h)$. We tied off the major branches and attached the endplates to the distal and the proximal ends with sutures and tissue adhesive (LiquiVet) and placed the segment into a tissue cage fixture, Figure 1(a). Circulating phosphate buffered saline (PBS) was used to ensure constant pressure (100 $\mathrm{mmHg})$ as well as flow and to maintain the artery physiologically opened at $37^{\circ} \mathrm{C}$ (Fig. 1 (b)).

The IVUS catheter was introduced and advanced on a 0.014" guide wire and a complete automatic pullback was performed from the distal to the proximal side. We repeated the experiment using human blood instead of PBS. After imaging, the artery was pressure fixed with $10 \%$ buffered formaldehyde under $100 \mathrm{mmHg}$ during three hours. After decalcification, the histology blocks were cut from distal to proximal every $2 \mathrm{~mm}$ (corresponding to 120 frames of the IVUS pullback) using the side rods. Blocks were then embedded in paraffin. For each block, three sections were used for histology. The first two sections were stained with hematoxylin and eosin (H\&E) and Movat Pentachrome, and the last section was kept unstained for additional future staining (e.g., Sirius Red). Hematoxylin and eosin are among the most commonly used stains in histopathology. Hematoxylin turns nuclei blue; eosin turns the cytoplasm (mostly composed of proteins arginine and lysine) pink. Another stain, the Movat pentachrome, colors cytoplasm in red, elastin fibers in black, collagen and reticulum fibers in yellow to greenish and proteoglycans in blue. Clear areas might represent water, carbohydrate, lipid, gas or decalcified areas. Sirius red, on the other hand, has been used exclusively for collagen staining in cardiovascular histopathology. Collagen fibers are stained red and can be distinguished morphologically from other tissue components in the plaque. 


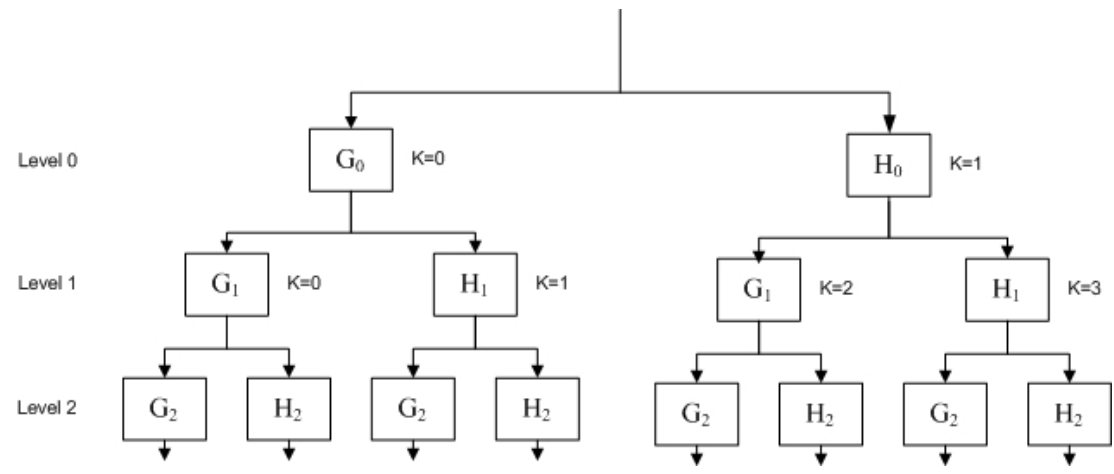

Figure 2. Tree structure for discrete wavelet packet frames (DWPF) and associate indexes. G and $\mathrm{H}$ are the highpass and lowpass filters, respectively.

The in-vivo data were collected during a routine cardiac intervention procedure with the abovementioned acquisition specifications and stored for offline processing.

\section{3- METHODS}

The discrete wavelet packet transform (DWPT) ${ }^{11}$ is the over complete version of the decomposition process in the discrete wavelet transform (DWT) ${ }^{12,13}$, in which every generated coefficient in the decomposition tree is passed through a high-pass and low-pass filters. The cost of such a generalization is an increase in computational complexity. Unlike the DWT and DWPT the decompositions are translation invariant in DWPF and no decimation occurs between levels, (Fig. 2). This makes the DWPF method superior for texture segmentation. Since the IVUS signals are sampled at the rate of $f_{s}$, it is more appropriate to consider the discrete signals that can be represented by a set of wavelet packet coefficients at the first scale $(l=0)$. For the tree-structured scheme demonstrated in Fig. 2, we can write:

$$
S_{2 k}^{l+1}(\omega)=G_{l}(\omega) S_{k}^{l}(\omega), S_{2 k+1}^{l+1}(\omega)=H_{l}(\omega) S_{k}^{l}(\omega)
$$

where $S_{k}^{l}(\omega)$ is the Fourier transform of the frame coefficients at channel $k$ and level $l$. The $G_{l}(\omega)$ and $H_{l}(\omega)$ are the highpass and lowpass filters at level $l$, respectively.

\subsection{Filter bank specification}

The highpass, $G_{l}(\omega)$, and lowpass, $H_{l}(\omega)$, filters at level $l$ can be written as ${ }^{9}: G_{l}(\omega)=G_{0}\left(2^{l} \omega\right)$. Consequently, the multi-channel wavelet schematic in Figure 2 behaves like a filter bank with channel filters $\left\{F_{k}^{l}(\omega) \mid 0 \leq k \leq 2^{l}-1\right\}$, where $F_{k}^{l}(\omega)$ can be derived recursively as follows:

$$
\begin{aligned}
& F_{0}^{0}(\omega)=G_{0}(\omega), F_{1}^{0}(\omega)=H_{0}(\omega) \\
& F_{2 k}^{l+1}(\omega)=G_{l+1}(\omega) F_{k}^{l}(\omega)=G_{0}\left(2^{l+1} \omega\right) F_{k}^{l}(\omega) \\
& F_{2 k+1}^{l+1}(\omega)=H_{l+1}(\omega) F_{k}^{l}(\omega)=H_{0}\left(2^{l+1} \omega\right) F_{k}^{l}(\omega)
\end{aligned}
$$

It has been shown that the selection of the filters $G_{0}(\omega)$ and $H_{0}(\omega)$ can have significant impact on texture classification performance ${ }^{8}$. The filter candidates must satisfy crucial criteria such as symmetry as well as boundary accuracy and have optimal frequency response. Hence, we selected Lemarie-Battle ${ }^{13}$ wavelets that are symmetric (have linear phase response) and quadrature mirror filters (QMF). The former property alleviates boundary effects through simple methods of mirror extension. The wavelets using QMF as well as constructed filter bank $\left\{F_{k}^{l}(\omega)\right\}$ cover the entire frequency domain and satisfy the following property: 


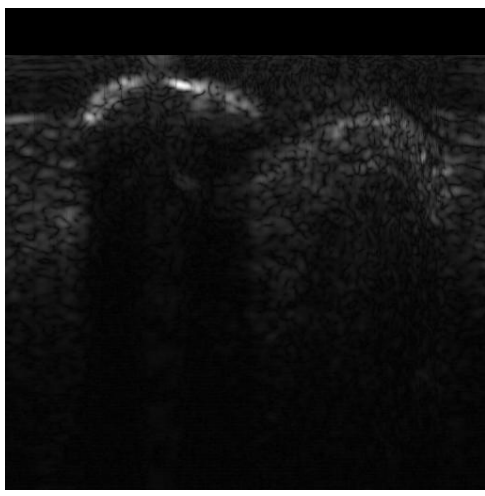

(a)

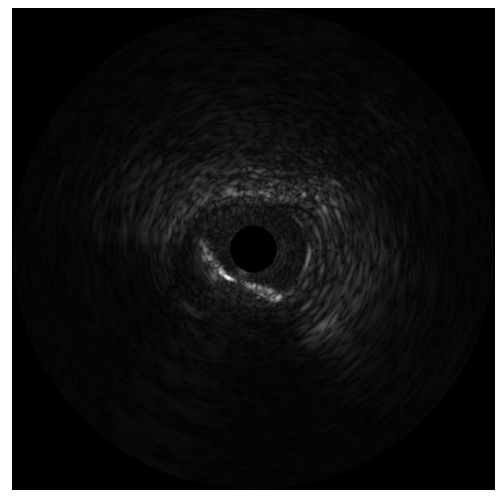

(b)

Figure 3. An IVUS frame, $(\mathrm{r}, \theta)$ domain $(\mathrm{a})$, scan converted Cartesian $(\mathrm{x}, \mathrm{y})$ domain, (transducer at center) (b)

$$
\begin{aligned}
& \left|G_{0}(\omega)\right|^{2}+\left|H_{0}(\omega)\right|^{2}=\left|G_{0}(\omega)\right|+\left|H_{0}(\omega)\right|=1 \\
& \sum_{k=0}^{2 l-1}\left|F_{k}^{l}(\omega)\right|^{2}=\sum_{k=0}^{2 l-1}\left|F_{k}^{l}(\omega)\right|=1
\end{aligned}
$$

\subsection{Feature extraction}

The features were extracted by processing of the IVUS signals for every frame in the $(r, \theta)$ domain. Each frame contains 256 lines that span over 360 degrees with 2048 samples per line. In order to have an optimized frame size with respect to the computational complexity and the textural resolution, we interpolated (bilinear) and decimated the signals in the lateral and axial directions by a factor of 2 and 4, respectively, to generate the square $M \times M, M=512$ pixels frame. Figure 3 illustrates an IVUS image in $(\mathrm{r}, \theta)$ and Cartesian $(\mathrm{x}, \mathrm{y})$ domain. For each IVUS frame, a tensor product extension was used in which the channel filters were denoted by $F_{i \times j}^{l}\left(\omega_{r}, \omega_{\theta}\right)=F_{i}^{l}\left(\omega_{r}\right) F_{j}^{l}\left(\omega_{\theta}\right)$. In the 1-D case, the wavelet and scaling functions concentrated mostly on one half of the frequency axis; on the other hand, the resulting 2-D basis will concentrate mostly around one direction. Consequently, such an extension will lead to the orientation selectivity in the decomposition tree. Four possible orientations can be considered excluding the root node, which is omnidirectional.

1. The node last filtered by $G_{l}\left(\omega_{r}\right) H_{l}\left(\omega_{\theta}\right)$ corresponds to the vertical orientation. The highpass filter $G_{l}$ and lowpass filter $H_{l}$ are applied in the axial and lateral directions, respectively.

2. The node last filtered by $H_{l}\left(\omega_{r}\right) G_{l}\left(\omega_{\theta}\right)$ corresponds to the horizontal orientation. The lowpass filter $H_{l}$ and highpass filter $G_{l}$ are applied in the axial and lateral directions, respectively.

3. The node last filtered by $G_{l}\left(\omega_{r}\right) G_{l}\left(\omega_{\theta}\right)$ corresponds to the diagonal orientation. The highpass filter $G_{l}$ is applied in the axial and lateral directions, respectively.

4. The node last filtered by $H_{l}\left(\omega_{r}\right) H_{l}\left(\omega_{\theta}\right)$ has the same orientation as its parent. The lowpass filter $H_{l}$ is applied in the axial and lateral directions, respectively.

Finally, the envelope of the signals was computed ${ }^{8}$ and the feature matrix was constructed as follows:

$$
\vec{V}_{i, j}=\left\{e_{k, i, j}^{l} \mid 0 \leq k \leq\left(2^{l}-1\right), i, j=1, \ldots, M\right\}
$$

where $e_{k, i, j}^{l}$ represents the envelope value of pixel $(i, j)$ for the $k$-th component at level $l$. 


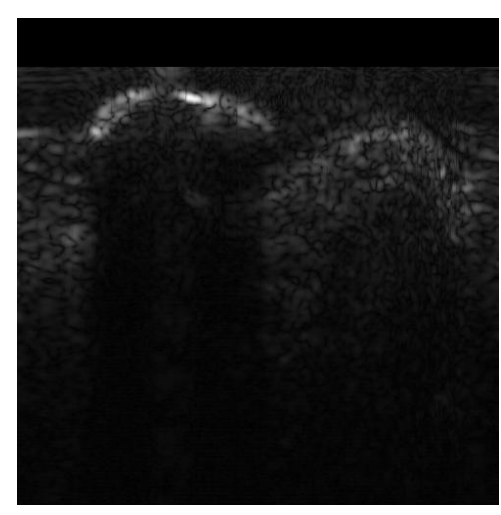

(a)

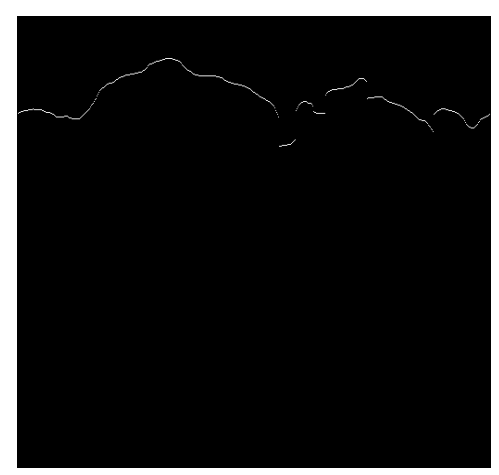

(c)

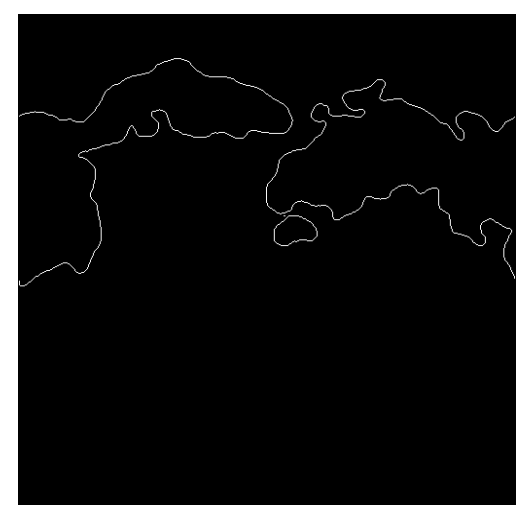

(b)

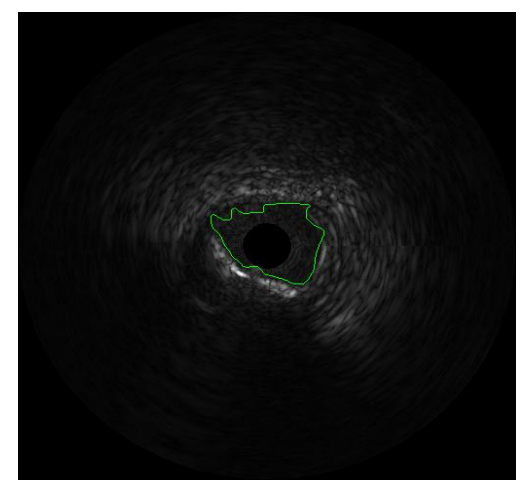

(d)

Figure 4. The original IVUS image in the $(\mathrm{r}, \theta)$ domain (a), The segmented image using DWPF using Lemarie-Battle filter of order $18, l=4, N_{c}=2$ (b), The closest set of pixels to the surface of the transducer (c), Lumen border after scan conversion (Cartesian domain) and spline interpolation (d).

\section{4- BORDER DETCTION METHODOLOGY AND RESULTS}

For every representation matrix $X_{M \times M}$ that contains feature vectors, a label was assigned to every pixel by modulo $N_{c}$. We computed the center of clusters $\left\{\vec{C}_{\kappa} \mid 0 \leq \kappa \leq N_{c}-1\right\}$ by calculating the mean vector for each class. The pixel $\left\{x_{i, j} \mid i, j=1, \ldots, M\right\}$ was assigned to the class $\kappa$, if the Euclidean distance between the corresponding pixel and the class center $\vec{C}_{\kappa}$ was the closest. The centers of the clusters were updated in an iterative fashion by recomputing the relative mean vectors. The procedure was terminated once no change in labeling occurred. Figure 4(b) demonstrates the segmented results after edge detection. In order to find the majority of pixels that lie on the lumen border, we identified the closest set of pixels $\overrightarrow{\mathbf{r}}$ in respect to the transducer surface and removed the outliers as follows:

$$
\begin{array}{r}
\overrightarrow{\mathbf{r}}=\left\{\bigcup_{i=1}^{M}\left(r_{j}, \theta_{i}\right)=1 \mid r_{j}=\min \left(r_{k}\right), k=1, \ldots, M\right\} \\
\mu=E[\mathbf{r}], \quad \sigma^{2}=E\left[(\mathbf{r}-\mu)^{2}\right] \\
\overrightarrow{\mathbf{r}}_{\text {ouliers }}=\left\{r_{j}|| r_{j}-\mu \mid>2 \sigma\right\}
\end{array}
$$

where $E, \mu$ and $\sigma^{2}$ denote the expectation, mean and variance, respectively. The resulting set of pixels has been illustrated in Figure 4(c). Finally, all the detected points are transformed into the Cartesian domain followed by an iterative spline interpolation until the closed form of contour is achieved, Figure 4(d). 


\subsection{Experimental results and quantification}

As we mentioned in Section 1, none of the existing algorithms is able to characterize the atherosclerotic tissues and differentiate between blood and plaque signals (detect the lumen border) simultaneously. Our results show that the proposed algorithm can classify tissues and detect the lumen border reliably. Previously we used 64 cross section of interest (CSI) collected from thirty two cadaver hearts, including $26 \mathrm{LAD}, 28 \mathrm{RCA}$ and $20 \mathrm{LCX}$ segments ${ }^{10}$. The overall classification performance was reported by two independent histopathologists to be $81.71 \%, 82.76 \%$ and $85.51 \%$ for fibrotic, lipidic and calcified regions, respectively. Figure 5 demonstrates the IVUS CSI, corresponding Movat Pentachrome histology image, and the IVUS-histology color map (IV-HCM). The blue, yellow and pink colors denote calcified, fibrotic, and lipidic plaque components, respectively.

We evaluated the algorithm performance on border detection using 18 in-vivo CSI collected from three in-vivo patients and 14 in-vitro CSI collected from two cadaver hearts. Figure 6 demonstrates the automatic detected lumen contours and the manually traced contours by an expert for both cases. Our results show that the manual and automated detected lumen borders are correlated very well $(r=0.9854, y=0.953 x+0.725)$, Figure $7(\mathrm{a})$. We also computed the Tanimoto coefficient $(\eta)$ to measure how well the automated detected contour is matched with corresponding manually traced contour

$$
\eta_{\text {contour }}=\frac{N_{c}^{\text {contour }}}{N_{a}^{\text {contour }}+N_{m}^{\text {contour }}-N_{c}^{\text {contour }}}
$$

where, $N_{a}, N_{m}$ are the number of enclosed pixels in automated and manually traced contours respectively and $N_{c}$ is the number of common pixels in both. Figure 6 shows the Box-Whisker plot of Tanimoto coefficients of automated and manually delineated lumen borders with length of 1.5 times interquartile range and confirms that the borders are matched reliably.

\section{5- SUMMARY AND CONCLUSION}

We employed a $40 \mathrm{MHz}$ single element mechanically rotating transducer and collected in-vivo and in-vitro RF data. In this paper, we presented a new processing technique based on multi-scale wavelet packets to differentiate between blood and non-blood signals on the IVUS images. The algorithm is also capable of classifying the atherosclerotic tissues into lipidic, fibrotic and calcified. Our proposed technique is unique in a sense that it is capable of detecting the lumen border and plaque components. The main advantage of the proposed algorithm is that it can reliably classify plaques and blood regardless of the transducer center frequency or spectrum while inconsistency among the alternative techniques employing spectrum-derived features within the transducer's bandwidth still remains a major challenge ${ }^{2}$.

The tissue classification accuracy had been evaluated to be $81.71 \%, 82.76 \%$ and $85.51 \%$ for fibrotic, lipidic and calcified regions, respectively. The tissue classification accuracy by IVUS virtual histology (IVUS-VH) ${ }^{1}$ technique using autoregressive (AR) analysis in combination with a classification tree for $30 \mathrm{MHz}$ single element transducers has been reported to be $90.4 \%, 92.8 \%, 90.9 \%$ and $89.5 \%$ for the training dataset and $79.7 \%, 81.2 \%, 92.8 \%$ and $85.5 \%$ for the test dataset in fibrotic, fibro-lipidic, calcified and calcified necrotic regions, respectively. We validated the performance of the algorithm for differentiating between blood and non-blood regions on IVUS images using 32 in-vivo and in-vitro CSI and the results demonstrated promise for reliable lumen border detection. The automated and manually detected lumen areas were highly correlated $(r=0.9854, y=0.953 x+0.725)$ and comparable to the results of the study conducted by Sonka et $\mathrm{al}^{5}$ using 38 in-vitro CSI at $30 \mathrm{MHz}(r=0.96, y=1.02 x+0.52)$.

However, a fully automated atherosclerotic tissue characterization algorithm is required to identify the vessel wall in addition to lumen border prior to tissue classification. We will examine the possibility of differentiation of vessel adventitia and plaque, or detection of internal elastic lamina, media and external elastic lamina in future studies. 


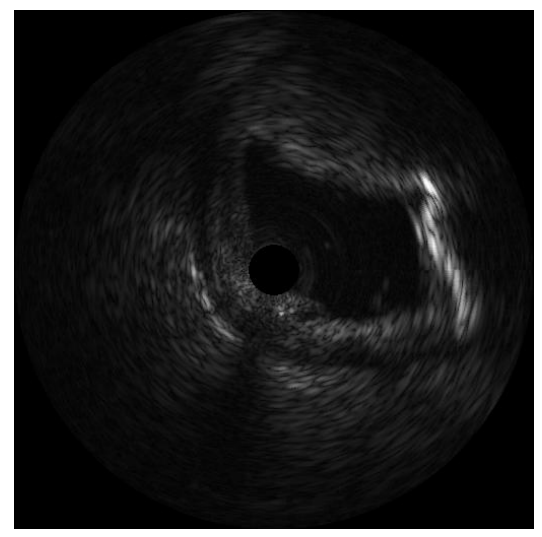

(a)

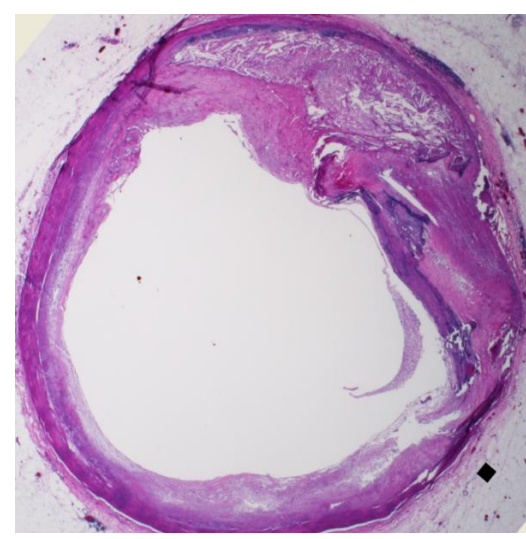

(b)

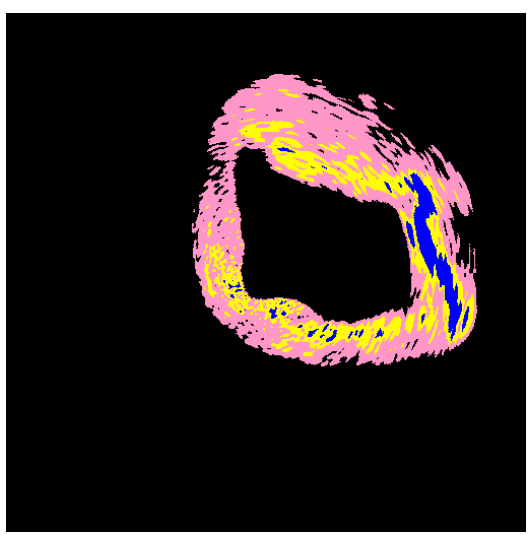

(c)

Figure 5. Movat Pentachrome histology image of cross section of interest (a) corresponding IVUS image (b), resulting IVUShistology color map generated by the algorithm using Lemarie-Battle filters of order 18, decomposition level $\mathrm{L}=2$, number of classes $\mathrm{N}_{\mathrm{c}}=4$ (c). Blue, yellow and pink colors represent calcified, fibrotic and fibro-lipidic tissues.

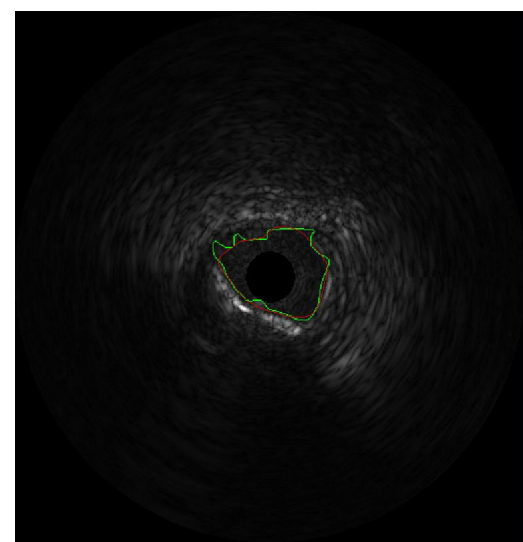

(a)

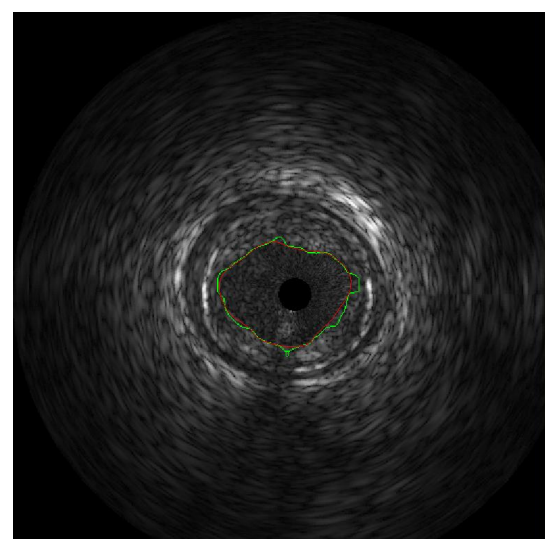

(b)

Figure 6. Automated detected lumen contour (green) by the algorithm using Lemarie-Battle filters of order 18, decomposition level $\mathrm{L}=4$, number of classes $\mathrm{N}_{\mathrm{c}}=2$ and manually traced contour (red) by and expert. In-vivo (a), In-vitro using circulating human blood (b)
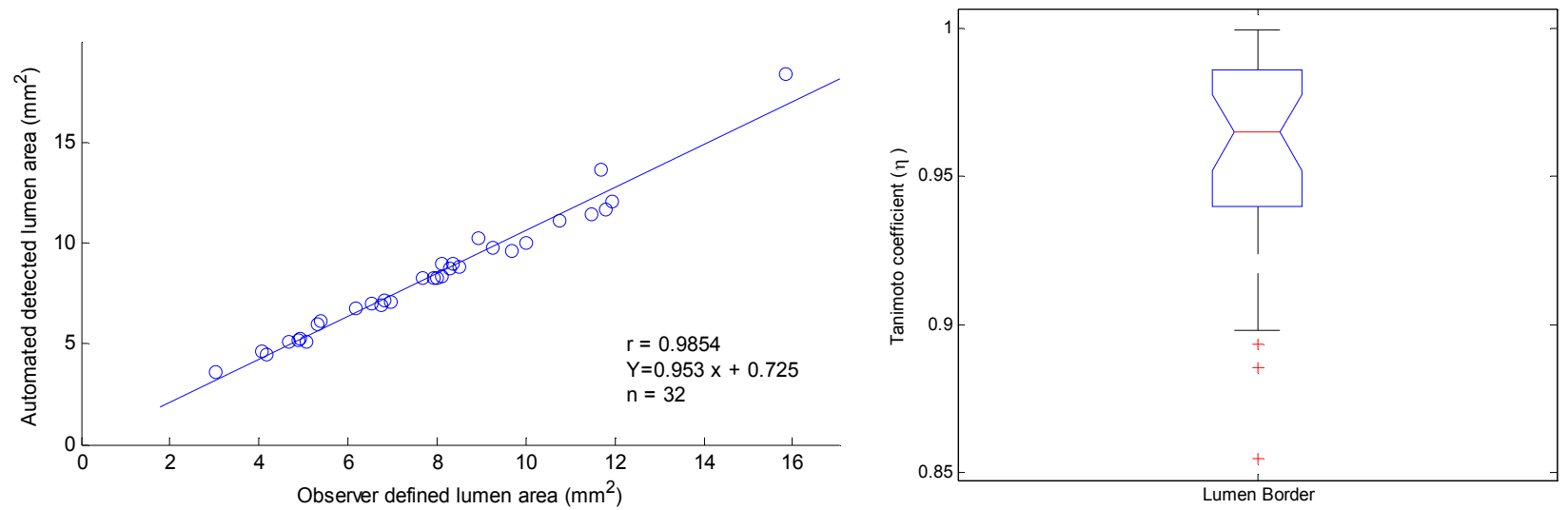

Figure 7. Comparison of automated detected lumen area and expert-defined lumen area (a), Box-Whisker plot of Tanimoto coefficients of lumen border. 


\section{ACKNOWLEDGEMENTS}

The authors would like to thank Boston Scientific Inc. (Fremont, CA) for providing the transducers and acquisition systems. We also thank Jennifer Lisauskas and Stephen Sum, at InfraRedx (Burlington, MA), who developed the artery fixation cage and their invaluable help in collecting IVUS data. The histology samples were processed at CVPath (Gaithersburg, MD) under the supervision of Dr. R. Virmani.

\section{REFERENCES}

[1] Nair, A., Kuban, B.D., Obuchowski, N., Vince, D.G., "Assessing spectral algorithms to predict atherosclerotic plaque composition with normalized and raw intravascular ultrasound data," Ultrasound in Med. \& Biol., vol. 27, no. 10, pp. 1319-1331, 2001 .

[2] Katouzian, A., Sathyanarayana, S., Baseri, B., Konofagou, E.E., Carlier, S.G., "Challenges in Atherosclerotic Plaque Characterization with Intravascular Ultrasound (IVUS): From Data Collection to Classification," IEEE Trans. on Information Technology in Biomedicine, Special issue on IVUS (in press).

[3] Kawasaki, M., Takatsu, H., Noda, T., Sano, K., Ito, Y., Hayakawa, K., Tsuchiya, K., Arai, M., Nishigaki, K., Takemura, G., Minatoguchi, S., Fujiwara, T., Fujiwara, H., ” In Vivo Quantitative Tissue Characterization of Human Coronary Arterial Plaques by Use of Integrated Backscatter Intravascular Ultrasound and Comparison With Angioscopic Findings," Circulation, vol. 105, no. 21, pp. 2487- 2492 May 2002.

[4] Virmani, R., Burke, A.P., Kolodgie, F.D., Farb, A., "Pathology of the thin-cap fibroatheroma: A type of vulnerable plaque," J. Inteven. Cardiol., vol. 16, No. 3, pp. 267-272, 2003.

[5] Sonka, M., Zhang, X., Siebes, M., Bissing, M.S., DeJong, S.C., Collins, S.M., Mckay, C.R., "Segmentation of intravascular ultrasound images; a knowledge-based approach," IEEE Tran. on Medical Imaging, vol. 14, no. 4, pp. 719-732, 1995.

[6] Meier, D.S., Cothren, R.M., Vince, D.G., Cornhill, J.F., "Automated morphometry of coronary arteries with digital image analysis of intravascular ultrasound," Am. Heart J., vol. 133, no. 6, pp. 681-690, 1997.

[7] Unal, G., Bucher, S., Carlier, S.G., Slabaugh, G., Fang, T., Tanaka, K., "Shape-driven segmentation of intravascular ultrasound images," CVII-MICCAI, pp. 50-57, 2006

[8] Laine, A.F., Fan, J., "Frame representation for texture segmentation,” IEEE Trans. Image Proc., vol. 5, no. 5, pp. 771-780.

[9] Rioul, O., Vetterli, M. "Wavelet and signal processing," IEEE Sig. Proc. Mag., pp. 14-38, Oct. 1991.

[10] Katouzian, A., Baseri, B., Konofagou, E.E., Laine, A.F., "Texture-driven coronary artery plaque characterization using wavelet packet signatures," submitted to IEEE Int. Symp. on Biomed. Imag. 2008, under review.

[11] Coifman, R.R., Wickerhauser, M.V., "Entropy-based algorithms for best basis selection," IEEE Trans. Inform. Theory, pt. II, vol. 38, no. 2, pp. 713-718, Mar. 1992.

[12] Rioul, O. “A discrete-time multiresolution theory,” IEEE Trans. Sig. Proc., vol. 41, no. 8, pp. 2591-2606, Aug. 1993.

[13] Mallat, S.G. "A theory of multiresolution signal decomposition: The wavelet representation," IEEE Trans. Patt. Anal. Mach. Intell., vol. 11, no. 7, pp. 674-693, 1989. 